\title{
Engineering and Technology Journal
}

Journal homepage: https://etj.uotechnology.edu.iq

\section{Image Processing Technique for Zinc Ion Sensing Using a Crystalline Fiber Sensor}

\author{
Omar S. Hassan a , Razi J. Al-azawi ${ }^{\text {b }}{ }^{\text {, Bushra R. Mahdi }{ }^{\circ} * \mathrm{c}}$ \\ ${ }^{\text {a,b }}$ Laser and Optoelectronics Dept., University of Technology-Iraq, Alsina'a street,10066 Baghdad, Iraq. \\ ${ }^{c}$ Ministry of Science and Technology, Laser and Optoelectronic center - Iraq, Bagdad Iraq. \\ *Corresponding author Email: boshera65m@gmail.com
}

\section{H I G H L I G H T S}

- Image processing was used with fiber to reduce the cost of work and increase the speed of obtaining results.

- The results of the image were translated into an intensity curve and drawn in the form of a force curve.

- The sensitivity of the sensor was obtained and found that it was equal to $73.47 \%$.

\section{A R T I C L E I N F O}

Handling editor: Ivan A. Hashim

Keywords:

PCF sensor

Fiber sensor

Image processing

Image processing with fiber sensor

Image Profile

Energy Distribution

\begin{abstract}
A B S T R A C T
In this paper, crystalline optical fibers were used as a sensor for sensing the zinc ion concentration using the image processing technique. The image of the laser spot transmitted through the optical fiber crystal sensor for each concentration of zinc ion solution. The sensor was made by welding a piece of LMA-10 crystal optical fiber from both ends of a single-mode optical fiber to obtain an SM-PCFSM type sensor. And it is possible to distinguish between one concentration and another by studying the change of the images obtained as a result of changing the concentration of the zinc ion. The sensitivity of the manufactured sensor was about $73.47 \%$.
\end{abstract}

\section{Introduction}

Microstructural fibers or optical fibers (PCFs), which are known as Holey fibers, have an array arrangement of microholes running in parallel along the entire length of the fiber. There are two types of photonic fibers, the first type is hollow core with an air clad around it in silica glass and the other is a solid core surrounded by an air clad in silica. The process of light transmission in the first type is based on the effect of the photon band gap (PBG) is the light-guiding mechanism of the second type, through which light is transmitted by the phenomenon of modulated total internal reflection (MTIR) to direct light [1]. There are elements in the environment that are considered heavy metals that have a dangerous impact on environmental pollution, as zinc is considered one of the elements polluting the environment when it exceeds the permissible levels in the environment [2].

Optical fiber sensor is one of the most interesting and advanced fields. Fiber sensor is day by day more attractive than other sensors, as it is immune to electromagnetic interference, high accuracy, needs no electrical source, can be operated remotely without contact, uncomplicated installation, explosion-proof, small size and light weight hence the importance of replacing fibers Optical sensors with other sensors [3][9][10][11].

Novelty in this paper is the use of image processing to analyze the results of laser spots resulting from environmental pollution with zinc ion using a PCF photonic fiber sensor using Mach Zehnder technique.

\section{Image processing}

An image can be described as a width and length area of pixels. It has a specific height and width calculated in pixels [5]. They are captured by optical devices such as mirrors, cameras, telescopes, binoculars, lenses, etc., [6]. In low-cost imaging and recent developments and increasing capabilities of hardware storage, there are increasing demands for high image quality in 
various types of applications including video and image processing [7]. The images were divided into multiple parts called image segmentation. It was commonly used to identify objects or other related details in digital images. Different ways lead to image fragmentation [8]. Image processing is based on one of the following methods:

1. Otsu's method from the threshold method.

2. K- means aggregation method by color-based segmentation.

3. Fragmentation by watershed.

4. Texture filtering.

\section{Practical Side}

The practical aspect included a basic part as can be seen in figure (Figure 1). The first included the manufacture of the optical crystal fiber sensor, or which was manufactured by welding a piece of crystal optical fiber with a length of $5.2 \mathrm{~cm}$ of type PCF-LMA_10 with a single-mode optical fiber on both sides. Welding optical fibers. The sensor is installed in a plastic case on both sides. Experimental setup block diagram of the practical aspect performed is shown in Figure (Figure 1).

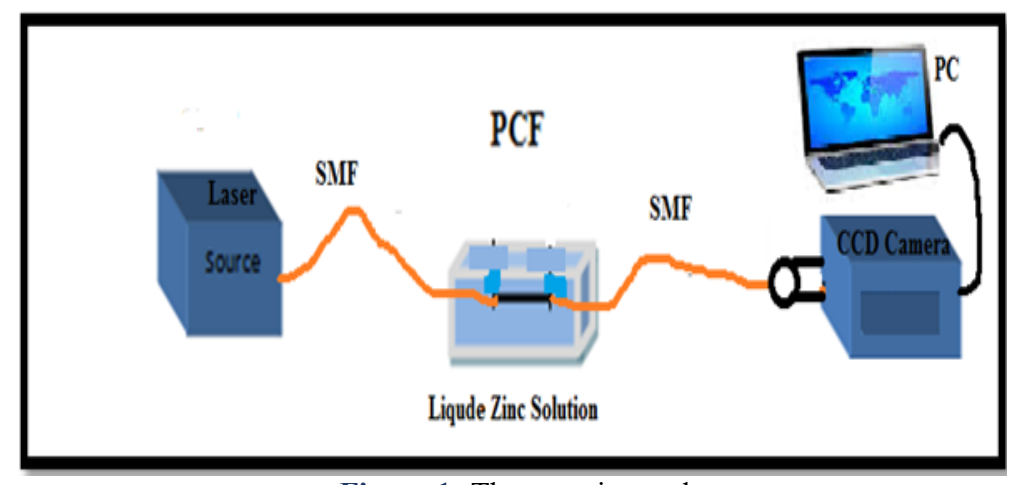

Figure 1: The experimental setup

The wavelength of the laser source is $450 \mathrm{~nm}$. The manufactured sensor is immersed in a reference sample of distilled water to take an image of the reference laser spot. CCD camera that was used with the following specifications: 20.7 MP, auto/manual Focusing, 10x optical zoom (24-240mm), OIS, Xenon and LED flash Spots obtained from the end side of the sensor far from the output laser source The cable is shown in Figure 5 (normal condition) and Figure 6. (Abnormal Status) These images are sent by a router to keep the room Closed and dark. These images are then processed using Image J. The sensitivity of the sensor was studied with decreasing concentration of zinc solution. The zinc solution was prepared with different concentrations in the range of $(1 \%, 2 \%$ and $3 \%) \mathrm{mg} / \mathrm{L}$, and the reference used was distilled water.

\subsection{Using image $J$ (version 1.8.0_172) for image processing}

To compute the percentage of the laser spot's affected pixels from the outside concentration, image processing was the best way to do that. An application developed at the National Institutes of Health and the Laboratory for Optical and Computational Instrumentation (LOCI, University of Wisconsin) using for image processing.

Image processing algorithm

1- $\quad$ Reading the Image.

2- Convert the image to gray scale.

3- $\quad$ From 2D image to 3D surface image.

4- Reading the image of the spot as a power curve.

\subsection{Results}

The laser distribution inside PCF LMA-10 surrounded with air and other concentration only can be seen with the CDD camera of smart phone.

The first step is to convert RGB images to 3D surface images as shown in figure (Figure 2) to compare the height of the entire image surface with different focus. This image shows the laser spot regions on the outer surface of the photonic fiber end that were surrounded by different concentrations of zinc solution compared with the front image, therefore, this image must first be read to calculate the total number of pixels of the unaffected regions. 


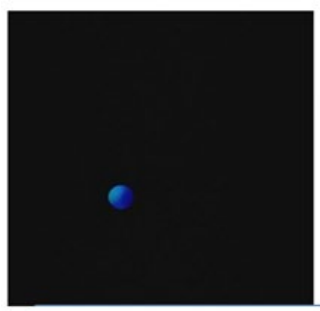

a

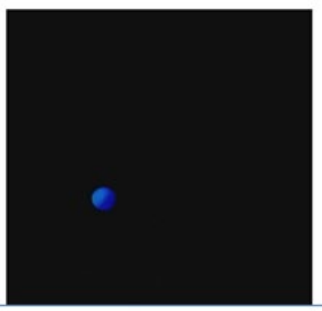

b

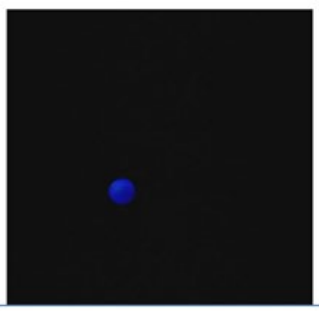

C

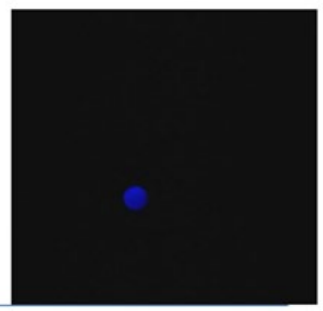

d

Figure 2: The spot of the output light with different concentration surrounded the PCF. (a) Output fiber sensor with immersed in distal water. (b)Output fiber sensor spot with $1 \%$ zinc concentration. (c) $2 \%$ zinc concentration. (d) $3 \%$ zinc concentration

\subsubsection{Convert the image into gray image}

After reading the image at first this image must be converted from RGB to Gray scale image then the gray scale image will be converted into shown as an information image. In this image white colored regions indicates the spot size and its affected part from the outside effects that surrounded the PCF, as shown in the following figure (Figure 3) it describes the gray scale images. It helps to calculate total number of pixels of unaffected regions of the laser spot.

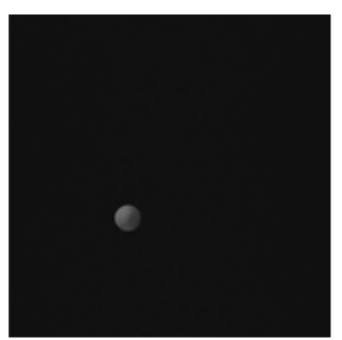

a

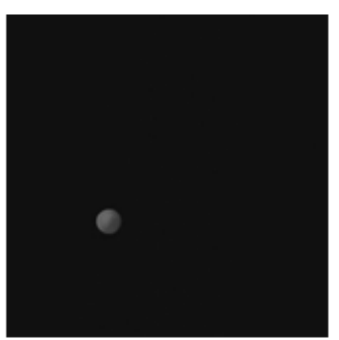

$b$

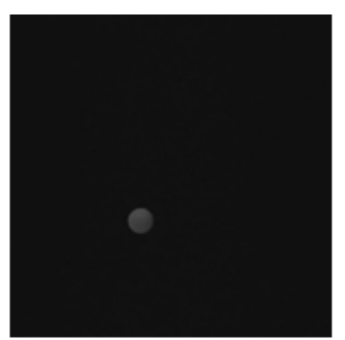

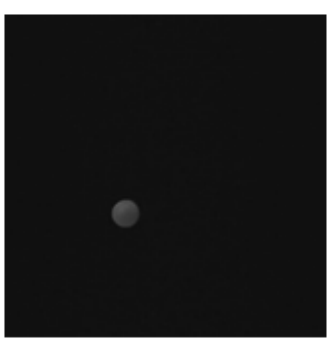

Figure 3: The gray scale of the spot of the output light with different concentration surrounded the PCF. (a) Output light with distal water. (b) spot image at $1 \%$ zinc concentration. (c) spot image at $2 \%$ zinc concentration. (d) spot image at $3 \%$ zinc concentration

\subsubsection{Convert the image into 3D surface image}

When different concentrations of immersed zinc were used for the fiber sensor, some different parameters were obtained with a change in the concentration surrounding the sensor.

The 3D surface of the laser spot image of the output fiber sensor with distal water is shown in figure (Figure 4). The 3D surface of the laser spot image of the output light with 1\% Zinc solution in water continuation is shown in figure (Figure 5). The 3D surface of the laser spot image of the output sensor with $2 \%$ Zinc solution in water continuation is shown in Figure (6).

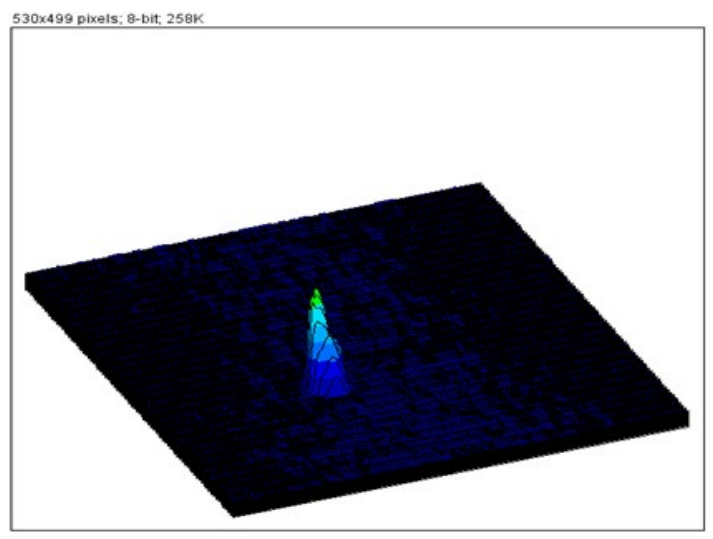

Figure 4: The 3D surface of the laser spot image of the output light with distal water surrounded the PCF sensor

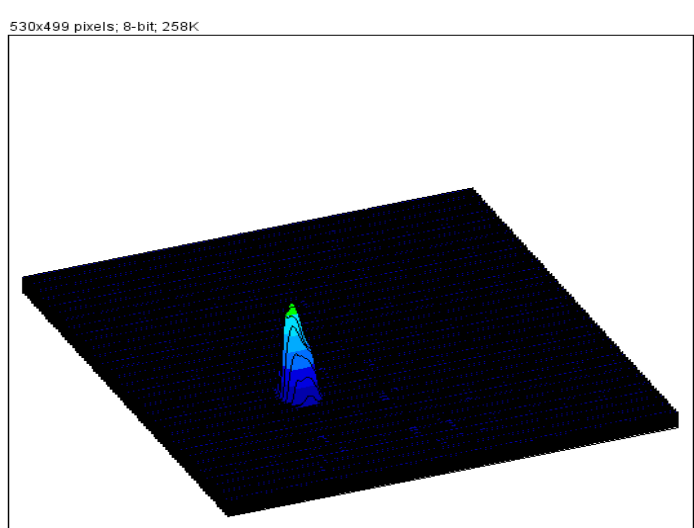

Figure 5: The 3D surface of the laser spot image of the output sensor with $2 \% \mathrm{Zn}$ concentration surrounded the PCF sensor 


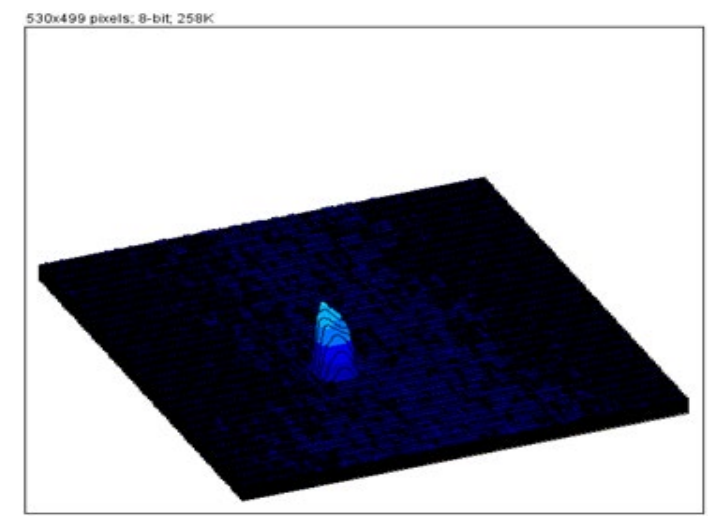

Figure 6: The 3D surface of the laser spot image of the output light with low concentration of $1 \% \mathrm{Zn}$ surrounded the PCF

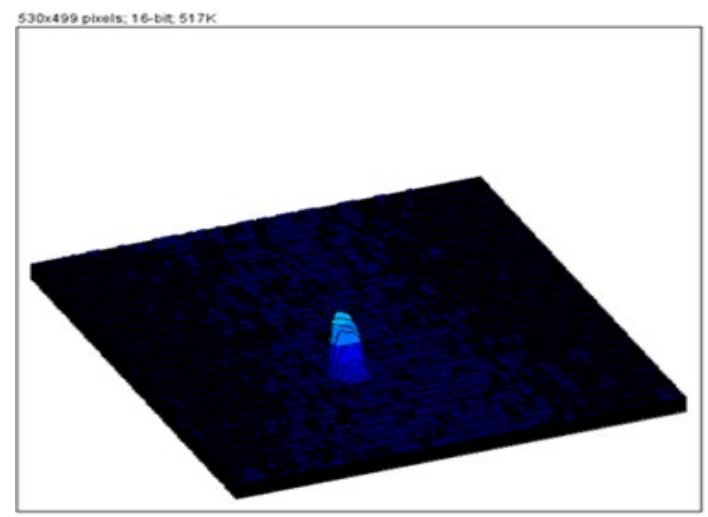

Figure 7: The 3D surface of the laser spot image of the output sensor with $3 \%$ concentration of $\mathrm{Zn}$ surrounded the PCF

The 3D surface of the laser spot image of the output sensor with 3\% Zinc solution in water continuation is shown in Figure (7). Converting the spot intensity in the images shown in Figure (8) above leads to a method for comparing light output in image processing using a CCD camera to capture the image and software to compare the results.

By comparing the results obtained, we find that there are differences in the intensity distribution of the laser radiation for each concentration compared to the source radiation without any external effects. These changes act like as sensor to the changes in the materials surrounding the PCFs. It is important to make the fiber sensitive to every change, no matter how small, to ensure quality and monitor changes.

The sensitivity of the sensor was obtained by taking the slop of the curve of the maximum intensity with the concentration, and we found that it was equal to $73.47 \%$.

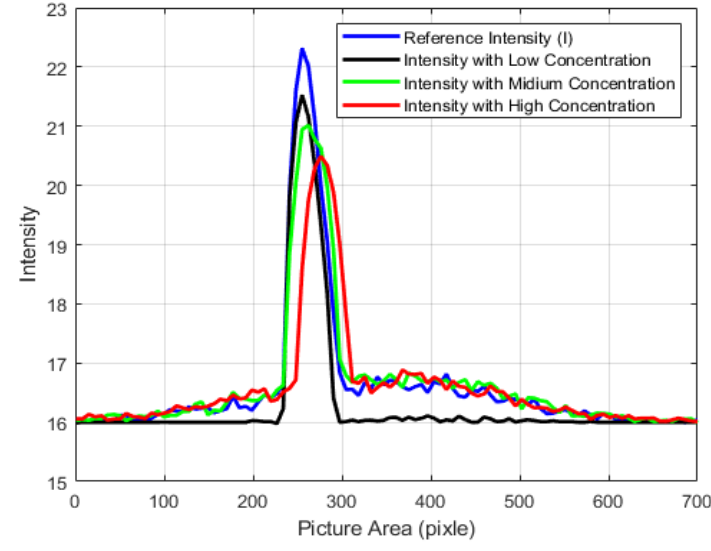

Figure 8: Translating the images of the laser spot of the output light to an intensity curve

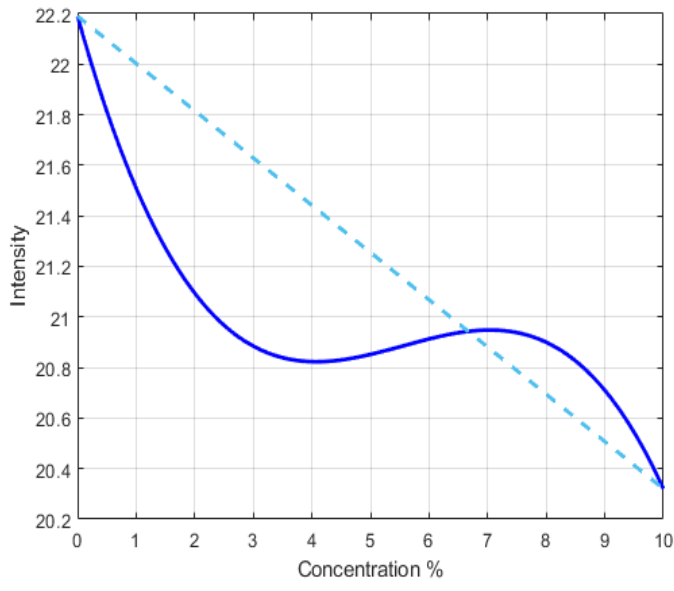

Figure 9: The relation between the concentration and the maximum intensity

\section{Conclusions}

PCF is used as a general sensor for contamination, and zinc is one of the important and variable factors that directly affect the wave outside the fibers as a result of direct adhesion to the fibers. The use of PCFs as a fiber sensor produces a new result that is useful in future works which leads to the development in the field of optical fiber sensor with high sensitivity sensors, and the high sensitivity of the fiber towards zinc enables its detection at levels close to change from the normal level, it is now possible to find Its concentration is higher than normal values. Image processing is not an acceleration in the work of fiber sensors without the need for complex devices to analyze the external signal and once it is translated into digital images and compared with the source and obtain clear results that can be calculated. The sensor proved highly sensitive in distinguishing between the concentration of zinc-contaminated solution with distilled water.

\section{Acknowledgment}

I extend my thanks to all the individuals and institutions that contribute to the development and support of scientific research from the university's technological inventors and their professors. I also extend my thanks to the Iraqi Ministry of Science and Technology represented by the Department of Material Science, Department of Laser Engineering.

\section{Author contribution}

All authors contributed equally to this work. 


\section{Funding}

This research received no specific grant from any funding agency in the public, commercial, or not-for-profit sectors.

Data availability statement

The data that support the findings of this study are available on request from the corresponding author.

Conflicts of interest

The authors declare that there is no conflict of interest.

\section{References}

[1] A. Z. Mohammed, B. R. Mahdi, L. K. Humdi, Photonic Crystal Fiber Magnetic Field Sensor Based on Ampere Force, Sci. Int., 29 (2017) 99-102.

[2] A. H. K. M. Bushra R. Mhdi, Nahla A. Aljabar, Suad M. Ali, Design and Construction Optical Fiber Sensor System for Detection the Stress and Fine Motion, Int. J. Adv. Appl. Sci., 2 (2013) 4-12. https://doi.org/10.11591/ijaas.v2i2.459

[3] A. Z. Mohammed, Photonic crystal fiber Mach-Zehnder interferometer pH sensing, AIP Conf. Proc., 2045 (2018) 020010. https://doi.org/10.1063/1.5080823

[4] S. R. A. Tahhan, A. Falih, Design and Simulation of Fiber Bragg Grating by Comsol Multiphysics Software, 6 (2017) 2085-2095.

[5] R. A. Kamil, K. H. Al-saedi, R. J. Al-azawi, A Novel Approach for Optic Disc Detection in RGB Retinal Fundus Images, Int. J. Sci. Res.,6 (2017) 1263- 1268. https://doi.org/10.21275/ART20175393

[6] Y. A. Alsultanny, Laser Beam Analysis Using Image Processing, J. Comput. Sci., 2 (2006) 109-113.

[7] M. M. Alkhabet, S. H. Girei, S. Paiman, N. Arsad, M. A. Mahdi, Highly Sensitive Hydrogen Sensor Based on PalladiumCoated Tapered Optical Fiber, at Room Temperature, Eng. Proc., 2 (2020)8. https://doi.org/10.3390/ecsa-7-08186

[8] K. Ahmed, B. K. Paul, F. Ahmed, A. Jabin, M. Shahin, Numerical demonstration of triangular shaped photonic crystal fibre-based biosensor in the Terahertz range, Inst. Eng. Technol. Optoelectron., 15 (2021)1-7. https://doi.org/10.1049/ote2.12006

[9] K. S. Shamna, Satellite image resolution and brightness enhancement using discrete, stationary wavelet and singular value decomposition, 2014 International Conference on Power Signals Control and Computations (EPSCICON), Thrissur, India, 2014, 1-4. https://doi.org/10.1109/EPSCICON.2014.6887496

[10] R. Islam ,M. Rafiqul, An Image Processing Technique to Calculate Percentage of Disease Affected Pixels of Paddy Leaf, Int. J. Comput. Appl., 123 (2015) 28-34. https://doi.org/10.5120/ijca2015905495

[11] R. Hani, B. R. Mahdi, A. Z. Mohammed, Photonic crystal fiber sensor for blood with different concentration of zinc, Mater. Sci. Forum, 1002 (2020) 290-299. https://doi.org/10.4028/www.scientific.net/MSF.1002.290 